\title{
Giant acrochordon of vulva: A rare occurrence
}

\author{
Agarwal $A^{1}$, Garg $C^{2}$, Mukherjee $S^{3}$, Mukherjee $S^{4}$, Kakkar $K P^{5}$ \\ ${ }^{1}$ Senior Resident, Department of General Surgery; ${ }^{2}$ Assistant Professor, Department of Pathology; \\ ${ }^{3}$ Associate Professor, Department of General Surgery; ${ }^{4}$ Associate Professor, Department of Obstetrics \\ \& Gynaecology; ${ }^{5}$ Professor and Head, Department of Pathology; Rohilkhand Medical College and \\ Hospital, Bareilly, U.P., India-243001
}

\begin{abstract}
Acrochordon, commonly called skin tag or fibroepithelial polyp is a benign mesenchymal lesion which is usually hyperpigmented and appears as surface nodule or papilloma on healthy skin. Most acrochordons vary in size from $2-5 \mathrm{~mm}$ in diameter, although larger acrochordons up to $5 \mathrm{~cm}$ in diameter are sometimes evident. The most frequent localizations are the neck and the axilla, but any skin fold including groin may be affected. Genital presentation in females has a greater predilection for the vulvovaginal region as compared to cervix. Diagnosis is mainly based on histopathology which is necessary not only to rule out malignancy but also to differentiate from other lesions like angiomyxoma which mimics fibroepithelial polyps. The case report is rarest of its kind with regard to its size as the polyp measured 20x16x12 cm and weighed approximately 3 kilograms. Thus, it becomes one of the largest acrochordons reported ever.
\end{abstract}

\section{Keywords: Acrochordon, Giant, Vulva}

\section{Address for correspondence}

Arjun Agarwal

Ashok-Kiran hospital, 35/C 8, Rampur Garden

Bareilly, Uttar Pradesh, India-243001

Phone: 918791865692

Email: arjunbly@hotmail.com

\section{Introduction}

Acrochordons are a type of mesenchymal lesion that typically occur in women of reproductive age. These polyps have a predilection for the vulvovaginal region. Infrequently they are found on cervix and rarely on extra-genital sites. These are hormone sensitive lesions. These are usually asymptomatic and do not become painful unless inflammation is present. Acrochordons typically do not grow larger than $5 \mathrm{~cm}$ in diameter and are usually incidental findings on gynecological examination. ${ }^{1}$ They can be polypoid or pedunculated and are usually solitary. Symptoms usually include bleeding, discharge and general discomfort with sensation of a mass. There are only a handful of cases of giant acrochordons reported in literature, of which vulvar are even rarer. This case is unique because the lesion measured $20 \mathrm{~cm}$ in its widest diameter, which is one of the largest sessile acrochordon reported till date. The largest acrochordon reported is of $48 \mathrm{~cm}$ in greatest dimension. ${ }^{2}$

\section{Case Report}

A 55 year old multiparous female presented in the outpatients department with complaint of swelling on her genital area for 6 months. To begin with, the swelling was of a size of small lemon that gradually increased to its current size. There was no history bleeding per vaginum, 


\section{Case Report}

trauma, sexually transmitted disease or any other medical or surgical history. Her menstrual history was unremarkable. On examination there was a huge swelling of about $20 \times 16 \times 12 \mathrm{~cm}$ on her left labia majora. Swelling was non tender, firm, sessile and skin over the swelling was nodular (Figure 1a).There was no increase in the size of the mass with valsalva manoeuvre. Incisional biopsy of one of the surface nodules was done which was suggestive of an acrochordon. Perilesional incision was given keeping away from labia minora. As the neck was sessile, it was divided using electrocautery till a short pedicle was reached that was joining the swelling to the labia majora, which was transfixed with silk suture and the swelling was divided distally. The defect left was closed primarily with intermittent vertical mattress suture using nylon monofilament and the specimen was sent for histopathological examination.

On gross examination, the large soft tissue mass weighed 2.85 kilograms with normal overlying skin which was thrown into variable sized polypoidal folds or nodules (Figure 1b). Cut section showed thinned out epidermal lining with deeper grey white areas. Microscopy revealed thinned out keratinised stratified squamous epithelium with underlying fibrovascular stroma. Stroma was edematous with few scattered stellate cells and several irregular shaped thin walled vessels and perivascular lymphocytic infiltrate (Figure 1c, d). A histopathological diagnosis of Acrochordon or Fibroepithelial polyp was ascertained without evidence of any malignant change.

Patient was discharged on second postoperative day and sutures were removed two weeks later. Patient has been followed for the last 5 months without any signs of recurrence.

\section{Discussion}

Acrochordons have an overall incidence of $46 \%$ with maximum occurrence in reproductive age group in females. ${ }^{1}$ Acrochordons have a rise in incidence through the fifth decade. By the age of 70 years, $59 \%$ of persons may have acrochordons. ${ }^{3}$

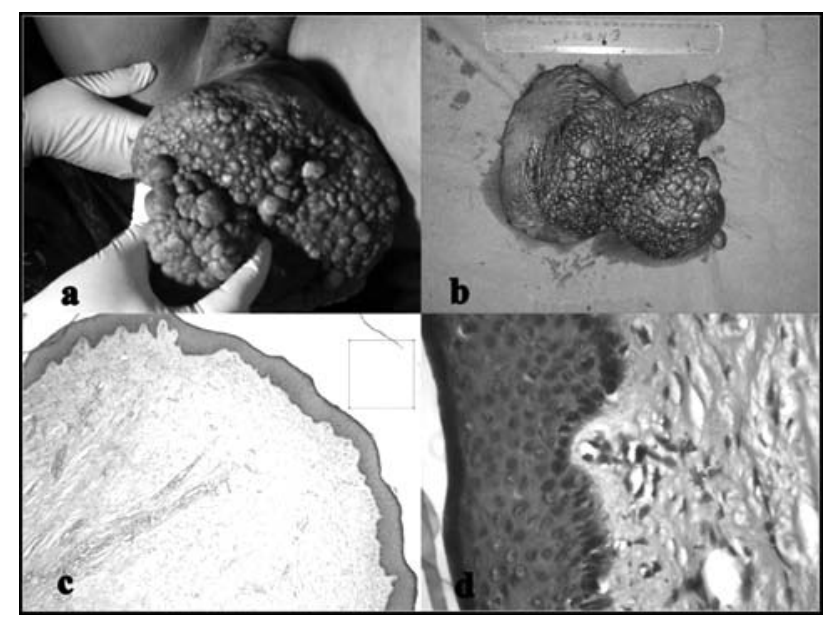

Figure 1: a) Shows a $20 \times 16 \times 12 \mathrm{~cm}$ swelling over left labia majora with nodular surface, $b$ ) Shows excised specimen of the swelling weighing $2.85 \mathrm{~kg}, \mathrm{c}$ ) Shows thinned out keratinised stratified squamous epithelium with underlying edematous fibrovascular stroma and scattered stellate cells (H\&E X 40x), d) Shows several irregular shaped thin walled vessels and perivascular lymphocytic infiltrate (H\&E X 400x).

Acrochordons are mostly skin coloured and pedunculated lesions that tend to occur in areas of skin folds. A family history may exist but tumors are usually asymptomatic. However, concerns may relate to a suspected sexually transmitted disease or risk of harbouring infections. But themselves, acrochordons are not infectious or malignant. In women, genital skin tags appear mostly in the vagina, rarely in the vulva. ${ }^{4}$

There are only a few reported cases of giant fibroepithelial stromal polyps of the vulva so far. ${ }^{5-}$ ${ }^{7}$ Occasionally reports have mentioned about recurrence of the lesion as well. ${ }^{6}$

Differential diagnosis that should be considered include Neurofibroma, Premalignant fibroepithelial tumour, Seborrheic keratosis, Angiomyxoma and genital warts. ${ }^{8}$

Skin tags are generally treated for cosmetic reasons. Small, pedunculated acrochordons may 


\section{Case Report}

be removed with curved or serrated blade scissors, cryotherapy or even laser surgery. Electrodesiccation is another option. ${ }^{9}$ Larger skin tags require excision followed by repair and cosmetic enhancement. ${ }^{10}$

\section{Conclusion}

Giant acrochordon of the vulval region is a rare benign tumor that can be misinterpreted as malignant owing to its wide range of morphological appearances and rapid growth. Hence, a clinical suspicion and pathological interpretation is critical for diagnosis as well as to exclude other neoplasms like angiomyxoma. Proper surgical treatment requires definite pathological diagnosis.

Acknowledgement- Our patient, who was very cooperative and gave her consent for the article.

\section{References}

1. Nucci MR, Vulvar Neoplasia. In: Nucci MR, Olivia E, editors. Gynecologic Pathology: A Volume in Foundations in Diagnostic Pathology Series. Ist ed. Elsevier Churchill Livingstone, 2009: p31-32.

2. Wallach SJ. Approach to the giant acrochordon. Female Pelvic Medicine \& Reconstructive Surgery 2010; 16: S34. http://dx.doi.org/10.1097/01.spv.0000370822.96583.e3

3. Schwartz RA, Terilkowrka A. Acrochordon,e. Medical Dermatology 2009; 29(4); 1-7.

4. J Carter, P. Elliott, and P. Russell. Bilateral fibroepithelial polypi of labium minus with atypical stromal cells. Pathology 1992; 24: 37-9.

5. Bozgeyik Z, Kockoc E, Koc M, Dagli AF. Giant fibroepithelial stromal polyp of the vulva: extended field of view ultrasound and computed tomographic findings. Ultrasound Obstet Gynecol 2007; 30: 791-2. http://dx.doi.org/10.1002/uog.5143

6. Orosz Z, Lehoczky O, Szoke J, Pulay T. Recurrent giant fibroepithelial stromal polyp of the vulva associated with congenital lymphedema. Gynecol Oncol 2005; 98:168-71. http://dx.doi.org/10.1016/j.ygyno.2005.01.020

7. Navada MH, Bhat PRB, Rao SV, Nagarathna G. Large Fibroepithelial Polyp of Vulva. Dermatological medicine 2011: 1-2. http://dx.doi.org/10.1155/2011/273181

8. RoberS, AgnieszkaT, WandaM. Acrochordon. Retrieved from . "accessed on 16 January 2015"

9. Choudhary ST. Treatment of Unusually Large Acrochordon by Shave Excision and Electrodesiccation. J Cutan Aesthet Surg 2008; 1: 21-2.

10. Ilango N, Jacob J, Gupta AK, Choudhrie L. Acrochordon-A Rare Giant Variant. Dermatol Surg Surgery 2009; 35:1804-5. http://dx.doi.org/10.1111/j.1524-4725.2009.01294.x 